\title{
Constant mean curvature one surfaces in hyperbolic 3-space using the Bianchi-Calò method
}

\author{
LEVI L. DE LIMA and PEDRO ROITMAN
}

Departamento de Matemática, Universidade Federal do Ceará

Campus do Pici, 60455-760 - Fortaleza, Brasil

Manuscript received on October 29, 2001; accepted for publication on November 27, 2001; presented by MANFREDO DO CARMO

\begin{abstract}
In this note we present a method for constructing constant mean curvature on surfaces in hyperbolic 3-space in terms of holomorphic data first introduced in Bianchi's Lezioni di Geometria Differenziale of 1927, therefore predating by many years the modern approaches due to Bryant, Small and others. Besides its obvious historical interest, this note aims to complement Bianchi's analysis by deriving explicit formulae for CMC-1 surfaces and comparing the various approaches encountered in the literature.
\end{abstract}

Key words: Constant mean curvature one surfaces, congruence of spheres, rolling of surfaces, Weierstrass representation.

\section{INTRODUCTION}

It is generally accepted that the theory of surfaces in hyperbolic 3-space $\mathbb{H}^{3}(-1)$ with constant mean curvature equal to one (CMC-1 surfaces, for short) started with a seminal paper by R. Bryant (Bryant 1987), where he derives a representation for such surfaces in terms of holomorphic data in analogy with the well-known Weierstrass representation for minimal surfaces in $\mathbb{R}^{3}$ (Lawson 1980).

After the appearance of Bryant's investigation, many other researchers contributed to the subject. For example, M. Umehara and K. Yamada (Umehara and Yamada 1993) refined substantially Bryant's approach and were able to construct a varied class of examples of CMC-1 surfaces, besides developing many interesting global aspects in the theory. On the other hand, A. J. Small (Small 
1994) reinterpreted CMC-1 surfaces in terms of twistors, providing in particular a Weierstrass formula for such surfaces involving algebraic operations on the derivatives up to second order of a pair $(f, g)$ of holomorphic functions (see (8) below).

Consensual as it may be, the above account is not entirely correct and the main purpose of this note is to stress that in Bianchi's Lezioni di Geometria Differenziale (Bianchi 1927), we may find a recipe for constructing CMC-1 surfaces from holomorphic data. However, the motivation for writing this note goes beyond this historical curiosity, for it seems that there are at least two other reasons for exhibiting this old method to a wider audience.

The first one is that the method allows one to start with an arbitrary holomorphic map $f=f(z)$ defined in a region $\Omega \subset \mathbb{C}$ and, elaborating upon Bianchi's ideas, to end up with explicit formulae for a CMC-1 surface. More precisely, if we use the upper half-plane model for $\mathbb{H}^{3}(-1)$ with Cartesian coordinates $\left(x_{1}, x_{2}, x_{3}\right)$ so that the boundary at infinity is given by $x_{3}=0$, we have

THEOREM 1.1. In the above situation, the parametrization of a CMC-1 surfaces in terms of $f$ is given by

$$
\begin{aligned}
& x_{1}=\operatorname{Ref}-\frac{\left|f^{\prime}\right|^{2} \operatorname{Re}\left(f^{\prime} z\right)+\frac{1+|z|^{2}}{2} \operatorname{Re}\left(\left(f^{\prime}\right)^{2} \bar{f}^{\prime \prime}\right)}{\left|f^{\prime}\right|^{2}+\operatorname{Re}\left(f^{\prime} \bar{f}^{\prime \prime} \bar{z}\right)+\frac{\left|f^{\prime \prime}\right|^{2}\left(|z|^{2}+1\right)}{4}}, \\
& x_{2}=\operatorname{Imf}-\frac{\left|f^{\prime}\right|^{2} \operatorname{Im}\left(f^{\prime} z\right)+\frac{1+|z|^{2}}{2} \operatorname{Im}\left(\left(f^{\prime}\right)^{2} \bar{f}^{\prime \prime}\right)}{\left|f^{\prime}\right|^{2}+\operatorname{Re}\left(f^{\prime} \bar{f}^{\prime \prime} \bar{z}\right)+\frac{\left|f^{\prime \prime}\right|^{2}\left(|z|^{2}+1\right)}{4}}, \\
& x_{3}=\frac{\left|f^{\prime}\right|^{3}}{\left|f^{\prime}\right|^{2}+\operatorname{Re}\left(f^{\prime} \bar{f}^{\prime \prime} \bar{z}\right)+\frac{\left|f^{\prime \prime}\right|^{2}\left(|z|^{2}+1\right)}{4}} .
\end{aligned}
$$

Moreover, this map $f$ has an immediate geometric interpretation: it is simply the parametrized hyperbolic Gauss map, or in other words, the expression for the hyperbolic Gauss map in terms of a local complex parameter $z$ on $\Omega$. In fact, and this is an important issue here, our formulae coincide with Small's if the pertinent transformation between models for $\mathbb{H}^{3}(-1)$ is carried out (see Section 3).

The second reason is that in his way toward the construction of CMC-1 surfaces, Bianchi translates to hyperbolic geometry the solution of a strictly Euclidean-geometric problem involving the rolling of a pair of isometric surfaces, thereby establishing a surprising linking between these two geometries.

This article is organized as follows. In Section 2 we review some classical concepts in order to describe the rolling problem in Euclidean geometry. In particular we derive, following Bianchi, the so called Calò's formulae. In Section 3 we relate the solution of the Euclidean problem involving rolling of surfaces of Section 2 to the construction of CMC-1 surfaces in $\mathbb{H}^{3}(-1)$ and we sketch the proof of Theorem 1.1 above, besides exhibiting some simple examples constructed via the Bianchi-Calò method. 


\section{CONGRUENCE OF SPHERES AND ROLLING OF SURFACES}

Here is the first classical concept we shall meet. A congruence of spheres is a smooth two-parameter family of spheres in $\mathbb{R}^{3}$, that we will suppose parametrized by coordinates $(u, v)$. To each such congruence we may associate a function $R=R(u, v)$, the radius function, describing the radii of the spheres in the congruence. We also assume that the vector function $\mathbf{X}=\mathbf{X}(u, v)$ describing the centers of the spheres defines a regular surface which we call the surface of centers.

Generically there are two surfaces, the so-called envelopes, associated to a given congruence. In effect, a point $p \in \mathbb{R}^{3}$ belongs to an envelope $\xi$ if $p \in S$ for some sphere $S$ in the congruence and moreover $T_{p} \xi=T_{p} S$. If a congruence of spheres has two distinct envelopes we then have a natural correspondence between their points, namely, points on distinct envelopes correspond if they are the contact points of the envelopes with a given sphere of the congruence. The next proposition gives the expression for the envelopes in terms of the unit normal vector $\mathbf{N}=\mathbf{N}(u, v)$ and the metric of the surface of centers.

Proposition 2.1. In coordinates $(u, v)$,

$$
\xi=\mathbf{X}-R\left(\Delta(\mathbf{X}, R) \pm \sqrt{1-\Delta_{1} R} \mathbf{N}\right)
$$

where

$$
\Delta(\mathbf{X}, R)=\left(R_{u} A_{11}+R_{v} A_{12}\right) \mathbf{X}_{u}+\left(R_{u} A_{21}+R_{v} A_{22}\right) \mathbf{X}_{v}
$$

and

$$
\Delta_{1} R=R_{u}^{2} A_{11}+2 R_{u} R_{v} A_{12}+R_{v}^{2} A_{22} .
$$

Here, the matrix $A=\left[A_{i j}\right]$ is the inverse of the matrix defined by the metric in the given coordinates.

We now describe the rolling of isometric surfaces. Consider a pair $(S, \tilde{S})$ of isometric surfaces in $\mathbb{R}^{3}$, and let $p \in S$ and $\tilde{p} \in \tilde{S}$ be points corresponding under the isometry. Suppose $S$ is fixed in space and consider the two-parameter family of positions of congruent copies of $\tilde{S}$ such that to each $p \in S$ we consider a rigid motion of $\mathbb{R}^{3}$ (call it $H_{p}$ ) sending $\tilde{p}$ to $p, T_{\tilde{p}} \tilde{S}$ to $T_{p} S$, and further adjusted so that the differential of the isometry composed with $H_{p}$ is the identity map. This two-parameter family of positions for copies of $\tilde{S}$ is called the rolling of $\tilde{S}$ over $S$. The surface $\tilde{S}$ is called the rolled surface and $S$ the support surface.

Now fix a point $O \in \mathbb{R}^{3}$ and consider its image under the two-parameter family of rigid motions associated to the rolling of $\tilde{S}$ over $S$. In the generic case, the motion of $O$ defines a surface $\Sigma$ called the rolling surface with respect to the satellite point $O$.

The crucial point now is that the two concepts introduced so far, namely congruence of spheres and rolling of surfaces, share a close relationship. More precisely, we have

Proposition 2.2. Given a rolling of $\tilde{S}$ over $S$ as above, the rolling surface $\Sigma$ can be viewed as an envelope of a congruence of spheres having $S$ as its surface of centers and the sizes of the corresponding line segments joining points of $\tilde{S}$ to $O$ as radii. 
We are now in a position to formulate the problem in Euclidean geometry whose solution will lead us, according to Bianchi, to a method for constructing CMC-1 surfaces in $\mathbb{H}^{3}(-1)$ :

Find pairs $(S, \tilde{S})$ of isometric surfaces such that, for a convenient satellite point $O$, the rolling surface $\Sigma$ is contained in a plane.

Surprisingly enough, assuming that the plane in question is $\left\{x_{3}=0\right\}$ and moreover that the satellite point $O$ is the origin of our coordinate system, this problem admits a neat solution in terms of an arbitrary holomorphic function $f=f(z)$. More precisely, the radius function $R$ is given by

$$
R=\frac{1+|z|^{2}}{2}\left|f^{\prime}(z)\right|
$$

and the solution to our problem, namely, the coordinates of $\tilde{S}$ and $S$ are respectively given in terms of the holomorphic data as

$$
\tilde{x}=\left|f^{\prime}(z)\right| \frac{z+\bar{z}}{2}, \quad \tilde{y}=\left|f^{\prime}(z)\right| \frac{z-\bar{z}}{2 i}, \quad \tilde{z}=\left|f^{\prime}(z)\right| \frac{|z|^{2}-1}{2},
$$

and

$$
x=\operatorname{Re} f(z), \quad y=\operatorname{Im} f(z), \quad z=\left|f^{\prime}(z)\right| \frac{|z|^{2}+1}{2} .
$$

The above expressions are called Calò's formulae since they have been originally published by B. Calò in 1899 (Calò 1899) in another context involving isometric surfaces.

\section{THE BIANCHI-CALÒ METHOD}

In the last section, starting with a holomorphic map $f$, we have determined a pair of isometric surfaces such that one of the envelopes of the associated congruence of spheres was a plane. Now, in principle we could also determine the second envelope of the congruence associated to the rolling, which is then also contained in the upper half-space. It can be shown that the correspondence between the envelopes of the congruence associated to the Calò's pair $(S, \tilde{S})$ considered in the last section is a conformal map. This is proved in Bianchi's Lezioni when he considers Darboux congruencies and is one of the ingredients in the proof of the following central result, also due to Bianchi.

THEOREM 3.1. To each pair $(\tilde{S}, S)$ of isometric surfaces such that the rolling surface $\Sigma$ of the rolling of $\tilde{S}$ over $S$ is a plane there corresponds a CMC-1 given by the second envelope of the associated congruence of spheres considered as a surface in the standard upper half-space model of $\mathbb{H}^{3}(-1)$.

For the proof, look at the spheres of our congruence as horospheres by using the upper halfspace containing the surface of centers as a model for $\mathbb{H}^{3}(-1)$. One may check that $z$ is a conformal parameter for both envelopes, and that $f$ is the corresponding expression for the hyperbolic Gauss 
map $G$, which is precisely the correspondence between the envelopes. On the other hand, $G$ is known to be conformal, exception made for totally umbilical surfaces, exactly when the surface is a CMC-1 surface (see (Bryant 1987) or (Bianchi 1927)), and this concludes the proof.

Although Bianchi indicates how one can find CMC-1 surfaces starting with an arbitrary holomorphic map $f$ via Theorem 3.1, he does not complete his analysis by deriving explicit formulae. However, this can be carried out very simply: we use Calò's formulae (5) and (7) to compute the surface of centers $S$ and the radius function $R$, then we calculate, by means of (2), the envelopes of this congruence of spheres, one of them being a piece of the plane $\{z=0\}$ and the other one being our CMC-1 surface given by (1). This ends the sketch of the proof of Theorem 1.1.

Remark 3.2. It is easy to check that $f=G \circ X$, where $X=(x, y, z)$ is given by (1) and $G$ is the hyperbolic Gauss map $G$ of the corresponding CMC-1 surface.

Now we briefly indicate how Small's result, obtained by using a heavy algebraic-geometric machinery, relates to the one presented here. Small works in the so-called hermitian model for $\mathbb{H}^{3}(-1)$ and introduces, via twistor theory, a Gauss transform $\Gamma_{\mathcal{S}}=(f, g)$ of a null curve $\mathcal{S} \subset$ $S L(2, \mathbb{C})$, where $S L(2, \mathbb{C})$ is the orientation preserving isometry group of $\mathbb{H}^{3}(-1)$, in terms of a pair $(f, g)$ of holomorphic functions. His main result is that $\mathcal{S}$ can be recovered from $\Gamma_{\mathcal{S}}$ after applying dualization. After doing this, one realizes that $\mathcal{S}$ is given by the map $\omega: M \rightarrow S L(2, \mathbb{C})$,

$$
\omega=\left(\begin{array}{cc}
\alpha & \beta \\
\gamma & \delta
\end{array}\right)=\left(\begin{array}{cc}
\left(f^{\prime}\right)^{1 / 2}-\frac{1}{2} f\left(f^{\prime}\right)^{-3 / 2} f^{\prime \prime} & f\left(\left(f^{\prime}\right)^{-1 / 2}+\frac{1}{2} g\left(f^{\prime}\right)^{-3 / 2} f^{\prime \prime}\right)-g\left(f^{\prime}\right)^{1 / 2} \\
-\frac{1}{2}\left(f^{\prime}\right)^{-3 / 2} f^{\prime \prime} & \left(f^{\prime}\right)^{-1 / 2}+\frac{1}{2} g\left(f^{\prime}\right)^{-3 / 2} f^{\prime \prime}
\end{array}\right)
$$

where $f^{\prime}=d f / d g$ and $f^{\prime \prime}=d^{2} f / d g^{2}$. The expression for the CMC-1 surface in terms of the hermitian model is given by $\omega \bar{\omega}^{t}$, but in order to compare this with (1) one has to perform the transformation to the upper half-space model. In terms of the entries of $\omega$, this is given by

$$
x_{1}+i x_{2}=\frac{\alpha \bar{\gamma}+\beta \bar{\delta}}{|\gamma|^{2}+|\delta|^{2}}, \quad x_{3}=\frac{1}{|\gamma|^{2}+|\delta|^{2}} .
$$

We now take $g=z$ and Small's $f$ to be our $f$. A straightforward computation yields the equivalence between the methods.

We illustrate the method by retrieving two well-known examples. First, if we take $f(z)=z^{2}$, substitution in (1), after writing $z=r e^{i \theta}$, yields

$$
\mathbf{X}=\left(-r^{2}(\cos 2 \theta) \frac{5 r^{2}+3}{7 r^{2}+1},-r^{2}(\sin 2 \theta) \frac{5 r^{2}+3}{7 r^{2}+1}, \frac{8 r^{3}}{7 r^{2}+1}\right) .
$$

This is a catenoid cousin.

Now let $f(z)=\ln z$. Again, substitution in (1) yields

$$
\mathbf{X}=\left(\ln r-2 \frac{\left(r-r^{-1}\right)}{\left(r+r^{-1}\right)}, \theta, \frac{4}{\left(r+r^{-1}\right)}\right) .
$$


Or, writing $r=e^{s}$,

$$
\mathbf{X}=\left(s-2 \tanh s, \theta, \frac{2}{\cosh s}\right)
$$

This is a ruled example.

\section{RESUMO}

Nesta nota apresentaremos um método para construir superfícies de curvatura média constante um no 3espaço hiperbólico, a partir de funções holomorfas. Tal método foi introduzido nas Lezioni di Geometria Differenziale de Bianchi em 1927, antecedendo, portanto, em muitos anos, os pontos de vista mais modernos de Bryant, Small e outros. Além do seu óbvio interesse histórico, o objetivo da nota é complementar a análise de Bianchi, obtendo fórmulas explícitas para as superfícies de curvatura média constante um, e comparar os vários pontos de vista encontrados na literatura.

Palavras-chave: Superfícies de curvatura média constante um, congruência de esferas, rolamento de superfícies, representação de Weierstrass.

\section{REFERENCES}

Bianchi L. 1927. Lezioni di Geometria Differenziale, Terza Edizione, Nicola Zanichelli Editore, Bologna.

BRYANT R. 1987. Surfaces with constant mean curvature one in hyperbolic space, Astérisque 154-55: 321-347.

CALò B. 1899. Risoluzione di alcuni problemi sull'applicabilità delle superficie, Annali di Matematica IV.

Lawson HB. 1980. Lectures on minimal submanifolds I. Mathematics Lecture Series 9, Publish or Perish.

Small AJ. 1994. Surfaces of constant mean curvature one in $\mathbb{H}^{3}$ and algebraic curves on a quadric, Proc. of the A.M.S. 122: 1211-1220.

Umehara M and Yamada K. 1993. Complete surfaces of constant mean curvature 1 in the hyperbolic 3-space, Ann. of Math. 137: 611-638. 